ARTICLE

\title{
Highly efficient photoelectric effect in halide perovskites for regenerative electron sources
}

Fangze Liu', Siraj Sidhik ${ }^{2,3}$, Mark A. Hoffbauer ${ }^{1}$, Sina Lewis (10 1, Amanda J. Neukirch', Vitaly Pavlenko', Hsinhan Tsai (1) 1, Wanyi Nie (1) 1, Jacky Even (1) 4, Sergei Tretiak (10 1, Pulickel M. Ajayan (1) ${ }^{3}$, Mercouri G. Kanatzidis ${ }^{5,6,7}$, Jared J. Crochet (1D ${ }^{1}$, Nathan A. Moody ${ }^{1}$, Jean-Christophe Blancon (i) ${ }^{2 \bowtie} \&$ Aditya D. Mohite (1D 2,3凶

Electron sources are a critical component in a wide range of applications such as electronbeam accelerator facilities, photomultipliers, and image intensifiers for night vision. We report efficient, regenerative and low-cost electron sources based on solution-processed halide perovskites thin films when they are excited with light with energy equal to or above their bandgap. We measure a quantum efficiency up to $2.2 \%$ and a lifetime of more than $25 \mathrm{~h}$. Importantly, even after degradation, the electron emission can be completely regenerated to its maximum efficiency by deposition of a monolayer of $\mathrm{Cs}$. The electron emission from halide perovskites can be tuned over the visible and ultraviolet spectrum, and operates at vacuum levels with pressures at least two-orders higher than in state-of-the-art semiconductor electron sources.

\footnotetext{
${ }^{1}$ Los Alamos National Laboratory, Los Alamos, NM, USA. ${ }^{2}$ Department of Chemical and Biomolecular Engineering Rice University, Houston, TX, USA. ${ }^{3}$ Department of Material Science and Nanoengineering Rice University, Houston, TX, USA. ${ }^{4}$ Univ Rennes, INSA Rennes, CNRS, Institut FOTON - UMR 6082, 20, Avenue des buttes de Coesmes, Rennes, France. ${ }^{5}$ Department of Chemistry, Northwestern University, Evanston, IL, USA. ${ }^{6}$ Department of Materials Science and Engineering, Northwestern University, Evanston, IL, USA. ${ }^{7}$ Argonne-Northwestern Solar Energy Research (ANSER) Center, Northwestern

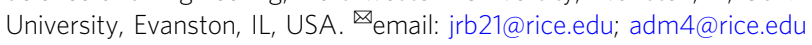


alide perovskites are now recognized as a new class of semiconductors with attractive properties for applications in optoelectronics, energy technologies, and photonics ${ }^{1,2}$. The materials abundance and low cost, ease of fabrication of single crystals or thin films using low temperature $\left(100^{\circ} \mathrm{C}\right)$ solution processing, tunability of their optical and electronic properties, and ability to carry relatively large charge current without achieving the level of crystal quality required in classic inorganic semiconductors (e.g. III-V, CdTe, $\mathrm{Cu}(\mathrm{In}, \mathrm{Ga}) \mathrm{Se}_{2}$ ) are the main reasons halide perovskites have drawn so much attention in the past few years. Although the main efforts in halide perovskite have been driven by record efficiencies in photovoltaics $(>25 \%)$, this class of semiconductors has recently seen a surge in the development of efficient proof-of-concept devices for diverse applications including light-emitting diodes, scintillators, $\mathrm{X}$-ray, and gamma radiation detectors ${ }^{3-9}$. Surprisingly, the figures of merit of halide perovskite-based devices have been on par with state-of-the-art inorganic semiconductor devices in several of these applications, which are consequences of the excellent intrinsic physical properties of halide perovskites as elucidated by several papers ${ }^{10-13}$

One of the most fundamental and technologically relevant phenomena in light-matter interaction is the demonstration of the photoelectric effect, where photons have been used to emit electrons in free space. Efficient electron emission from various types of materials has enabled technologies for many cutting-edge applications such as free-electron lasers, image intensifiers for night imaging, sources for next-generation electron-beam lithography, electron microscopy, and photomultipliers; it has also led to the elucidation of novel materials physics probed through the investigation of the spin, momentum, and energy of the emitted electron ${ }^{14-17}$. State-of-the-art electron sources (or photocathodes) are classified into three families depending on the active material type: metals $(\mathrm{Cu}, \mathrm{Mg}, \mathrm{Pb})$, alkali antimonide and tellurides $\left(\mathrm{Cs}_{2} \mathrm{Te}, \mathrm{K}_{2} \mathrm{CsSb}, \mathrm{Cs}_{3} \mathrm{Sb}\right)$, and III-V semiconductors $\left(\mathrm{GaAs}, \mathrm{GaN}\right.$, tertiary alloys of III-V materials) ${ }^{18}$. The differences between these photocathodes pertain to their figures of merit including their quantum efficiency (QE, defined as the number of free electrons emitted per excitation photon) and lifetime (usually defined as the time for the QE to degrade to $1 / \mathrm{e}$ of its initial value). For example, while metal-based electron sources exhibit excellent lifetime in operation (several months), their QE is typically of the order of $0.1 \%$ or less, which is more than two orders of magnitude lower than state-of-the-art semiconductorbased electron sources. On the other hand, the latter type of electron sources is more sensitive to degradation, and requires intensive material and device preparation ${ }^{18-21}$. The main advantage of non-metallic photocathodes is that they generate high free-electron currents (several milliamperes) due to their high QE spanning the ultraviolet and visible light spectrum range. Typically, QE larger than $10 \%$ can be achieved in both alkali- and III-V electron sources of high quality with crystalline perfect surfaces and usually after surface treatment that allows tunneling of electrons through the surface barriers and into vacuum ${ }^{18,21,22}$. For example, GaAs photocathodes achieve high QE by means of negative electron affinity (NEA) at their surface equivalent to surface dipoles pointing to the material bulk, which requires the deposition of an atomically thin layer of strongly electropositive alkali metal (usually Cs) on their surface and subsequent exposition to an oxidizing agent ${ }^{23,24}$.

Specifically, the semiconductor-based electron source technologies share stringent requirements in terms of material quality and require operation in ultra-high vacuum (typically $10^{11_{-}}$ $10^{-12}$ torr) with only traces of contamination elements such as $\mathrm{H}_{2} \mathrm{O}, \mathrm{O}_{2}, \mathrm{CO}_{2}{ }^{18,25-27}$. The latter impacts mainly the lifetime of the devices while the former determines the figures of merit of the electron sources including their QE. Thus, acting on one or both these constraints by reducing the complexity of the materials preparation, integration, and surface cleaning or by being able to operate the electron source at a higher pressure of vacuum will accelerate the development of such technologies through reducing the cost of fabrication and operational facilities, as well as by enabling electron sources with new technical and operational specifications. All of these have motivated the investigation of new classes of semiconductors for their potential use as easily replaceable, efficient, durable, low-cost, scalable, and adaptable (size, shape, and substrate) electron sources.

Here, we invoke Einstein's photoelectric effect, to report for the first time, efficient free-electron emission halide perovskite thin films operating in the visible to the ultraviolet spectral range. We measure a peak QE of $2.2 \%$ for the photoelectric effect and a lifetime exceeding $25 \mathrm{~h}$ under continuous operation and a vacuum pressure of $10^{-9}$ torr, which is more than two-orders higher than pressures typically used in state-of-the-art electron sources. We also show that electron emission can be completely regenerated to its maximum QE even after the perovskite electron source has almost completely degraded using an in-situ Cs deposition process. Finally, we show that by tuning the elemental composition of the halide perovskite films we can obtain electron emission across the entire visible spectrum. We anticipate that the observation of efficient electron generation using the photoelectric effect in halide perovskite thin films could pave the path for the development of a disruptive technology for realizing lowcost, high efficiency, and spectrally tunable electron sources based on halide perovskite semiconductors, which can be easily integrated and operated at much higher pressures of vacuum than their classic semiconductor-based counterparts without appreciable surface pre-treatments.

\section{Results}

Characterization of the photoelectric effect in halide perovskites. Halide perovskite thin films $\left(\mathrm{CsPbX}_{3}, X=\mathrm{Br}\right.$ or I) were made by mixing precursor powders $\left(\mathrm{PbX}_{2}\right.$ and $\left.\mathrm{CsX}\right)$ with the desired molar ratio in dimethyl sulfoxide or $\mathrm{N}$, $\mathrm{N}$-dimethylformamide and spin-coating on doped Si wafers. The sample preparation was made in a standard glovebox under $\mathrm{Ar}$ atmosphere to limit air contamination of the film's surface. The crystal structure of $\mathrm{CsPbX}_{3}$ along with the optical image of the films are presented in Fig. 1a. We characterized the absorbance, photoluminescence, and structure of both perovskite films in Fig. 1b, c (notice that $\mathrm{CsPbI}_{3}$ and $\mathrm{CsPbBr}_{3}$ respectively adopt the cubic and orthorhombic phases at room temperature) before loading them into an ultra-high vacuum chamber for photoelectric effect characterizations. The base pressure of the chamber was about $1 \times 10^{-9}$ torr with contaminant gas partial pressure below $10^{-11}$ torr, and the films were annealed at $350^{\circ} \mathrm{C}$ in the chamber to clean their surface from any organic or gas contaminant. We performed Auger electron spectroscopy (AES) on $\mathrm{CsPbBr}{ }_{3}$ before and after annealing, as shown in Supplementary Fig. 7. The magnitudes of characteristic Auger peaks from Cs $(49 \mathrm{eV}), \mathrm{Br}(55 \mathrm{eV})$, and $\mathrm{Pb}(97 \mathrm{eV})$ remained unchanged after UHV annealing. Moreover, we observe significant attenuation of the Auger signals associated with carbon $(275 \mathrm{eV})$ and oxygen $(510 \mathrm{eV})$. These results suggest that the chemistry of the perovskite thin films is not affected by UHV annealing while the latter process effectively removes oxygen adsorbed onto thin film surfaces, as well as amorphous carbon and solvent residue. The photoelectric effect was observed and studied by illuminating the sample with a monochromatic light beam and measuring the resulting photocurrent between the thin film sample and a counter anode while applying a potential difference between the 


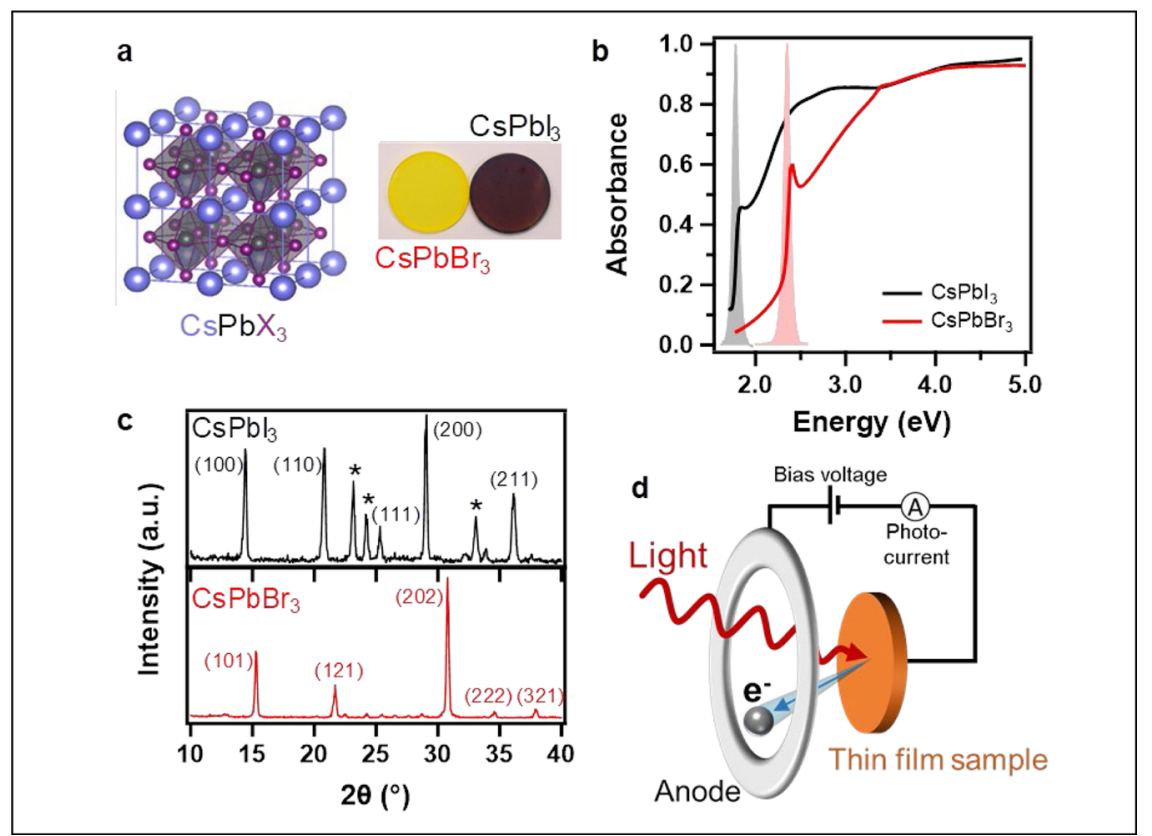

Fig. 1 Characterization of the halide perovskite thin films. a Cubic structure of the halide perovskites and picture of thin films on glass substrates. b Absorbance and photoluminescence (gray and red) spectra of the CsPbl3 and CsPbBr3 thin films. c Corresponding X-ray diffraction spectra. The stars indicate the presence of a residual non-perovskite yellow phase in CsPbl3. a.u. denotes arbitrary units. $\mathbf{d}$ Schematic of the photoelectric effect measurement setup. The films were prepared on 1 inch $^{2}$ silicon substrates for this measurement.

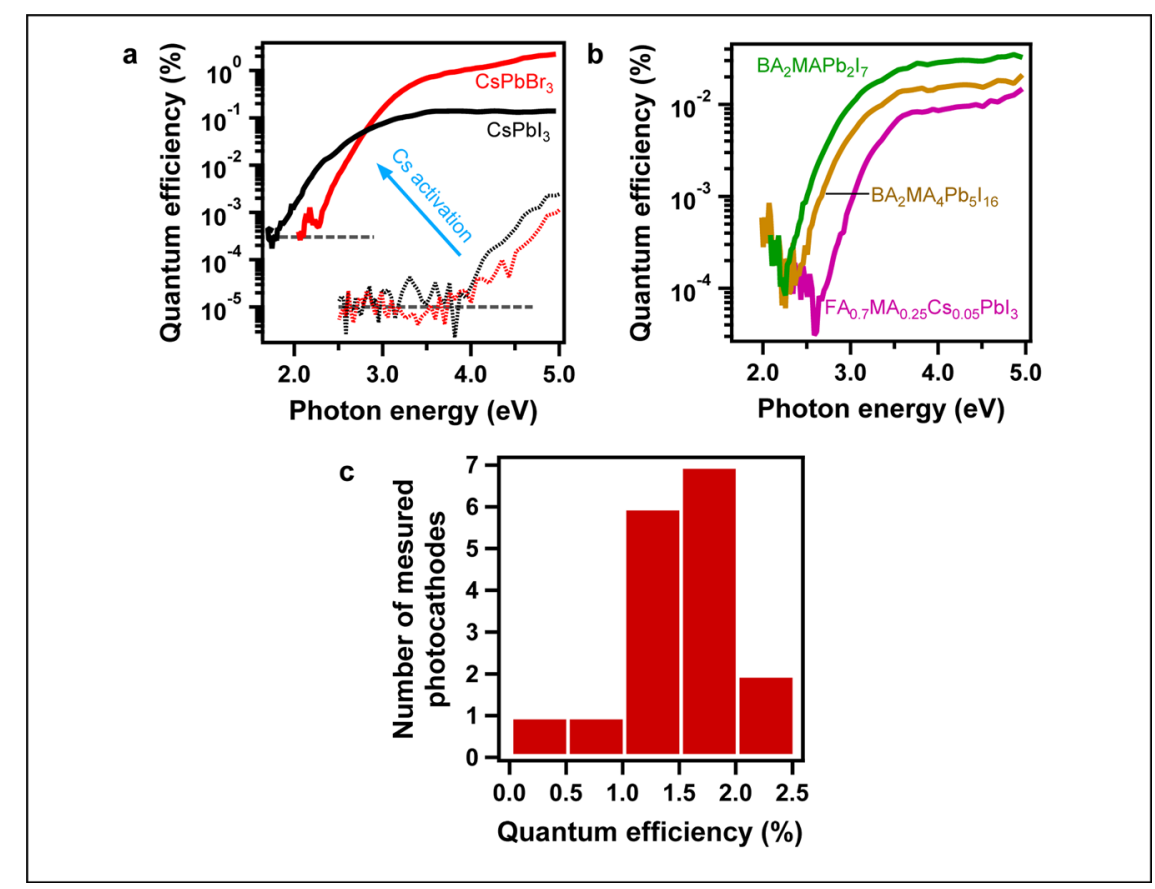

Fig. 2 Efficiency of the photoelectric effect in halide perovskites. a Quantum efficiency spectra of the photoelectric effect in the CsPbl3 and CsPbBr3 thin films before (dashed lines) and after (solid lines) Cs activation. Dashed gray horizontal lines indicate the emission threshold. b Same for the hybrid perovskite films: FA0.7MA0.25Cs0.05PbI3, BA2MA1Pb217, and BA2MA4Pb5l16. c Statistics of the maximum quantum efficiency performance measured for seventeen $\mathrm{CsPbBr} 3$ thin films.

two (Fig. 1d). The QE energy spectrum of the photoelectric effect was derived from the measurement of the photocurrent response as a function of the excitation light energy over the ultraviolet and visible range. The measurements were performed with and without the deposition in-situ of an ultra-thin layer (ideally one to a few monolayers) of Cs on the surface of the perovskite films.
Figure 2a illustrates the QE spectrum of the photoelectric effect for the $\mathrm{CsPbI}_{3}$ and $\mathrm{CsPbBr}$ thin films measured before (dashed curves) and after Cs deposition (solid curves). We observe a dramatic three to four orders of magnitude increase in the QE for all photon energies after the Cs deposition. Concomitant with the increase in the QE, we observe a lowering of the onset energy of 
the electron emission (i.e. the minimum photon energy necessary to observe the photoelectric effect) from $4.0 \mathrm{eV}$ to about the bandgap energy of $\mathrm{CsPbBr}_{3}(2.1 \mathrm{eV})$ and $\mathrm{CsPbI}_{3}(1.8 \mathrm{eV})$. The maximum $\mathrm{QE}$ values that were measured in the $\mathrm{CsPbBr}$ and $\mathrm{CsPbI}_{3}$ thin films are $2.2 \%$ at $5 \mathrm{eV}$ and $0.14 \%$ at $3.5 \mathrm{eV}$, respectively. In the $\mathrm{Cs} \mathrm{PbBr}_{3}$ films, the $\mathrm{QE}$ value remains greater than $1 \%$ for UV light with energy above $3.9 \mathrm{eV}$, and was measured to be larger than $0.25 \%$ at $3.1 \mathrm{eV}(400 \mathrm{~nm})$. These values are one order of magnitude smaller than the QE reported in the state-ofthe-art III-V semiconductor electron sources (e.g. GaN after Cs activation yields QE larger than $10 \%$ at $4.77 \mathrm{eV})^{18,21,22}$, which is rather impressive given the fact that III-V semiconductor electron sources involve complex and costly fabrication processes and surface pre-treatments in order to create high-quality materials with atomically perfect surfaces. In the case of halide perovskite thin films, the fabrication is simple and low cost, with the only demanding step being the Cs deposition. We also note that the QE performances of the halide perovskite films are more than two orders of magnitude better than metallic electron sources in the UV range and at a comparable vacuum level ${ }^{19}$. The reproducibility of the high QE observed in $\mathrm{CsPbBr}_{3}$ thin films was verified by testing 17 films yielding an average QE larger than $1.5 \%$ with two champion films exhibiting QE larger than $2 \%$ at $5 \mathrm{eV}$ as illustrated in Fig. 2c.

Next, we tested whether the Cs surface activation process was applicable to the broad family of organic-inorganic (hybrid) halide perovskites. The latter materials contain extremely reactive organic salts such as methylammonium (MA), butylammonium (BA), or Formadinium (FA), which replace the Cs atoms in the perovskite lattice (Fig. 1a). Three distinct hybrid perovskites that are used for fabricating state-of-the-art perovskite-based photovoltaic cells were tested: the $3 \mathrm{D}$ perovskite $\mathrm{FA}_{0.7} \mathrm{MA}_{0.25} \mathrm{Cs}_{0.05} \mathrm{PbI}_{3}{ }^{28}$, and the Ruddlesden-Popper layered hybrid perovskites $\mathrm{BA}_{2} \mathrm{MA}_{\mathrm{n}-1} \mathrm{~Pb}_{\mathrm{n}} \mathrm{I}_{3 \mathrm{n}+1}$ with $n=2$ or $5^{29}$. Here, we note that the hybrid perovskites were not annealed in an ultra-high vacuum because annealing these materials above $100^{\circ} \mathrm{C}$ is known to sublimate the organic cation and degrade the perovskites ${ }^{30}$. Despite these constraints, all the hybrid perovskite thin films demonstrated relatively efficient photoelectric effect with a low energy onset after Cs activation (Fig. $2 \mathrm{~b}$ and Supplementary Fig. 1). The emission onset for $\mathrm{BA}_{2} \mathrm{MA}_{1} \mathrm{~Pb}_{2} \mathrm{I}_{7}$ is close to its intrinsic bandgap, i.e. $2.35 \mathrm{eV}$ (photoelectric onset) versus $2.18 \mathrm{eV}$ (optical bandgap), where the difference between these two values could be accounted for by the binding energy of electron-hole pairs (excitons) ${ }^{31}$. However, the onset of electron emission was blue shifted in the other two hybrid perovskite thin films as compared to their bandgap energy. We speculate that this discrepancy is partly explained by our lack of control of the Cs activation process and the presence of absorbed gases on the film's surface (no annealing was done for hybrid perovskites), both of which can significantly affect the electronic structure on the surface of the perovskite films. The $\mathrm{QE}$ of the hybrid perovskites is of the order of $0.01 \%$ for photon energy between 3 and $3.5 \mathrm{eV}$, which is two-orders of magnitude higher than metallic electron sources in this energy range $\mathrm{e}^{18}$. These results attest to the robustness and potential of hybrid halide perovskites as electron sources despite the presence of reactive organic cations.

Understanding the physical origin of the photoelectric effect after Cesium activation. To further understand what limits the $\mathrm{QE}$ and what defines the onset photon energy of the electron emission in the halide perovskite thin films, we investigated the mechanism of the photoelectric effect, which consists of three basic steps (Fig. 3a): (i) absorption of photons in the bulk of the halide perovskite films and generation of photo-excited electrons in the conduction band, (ii) transport of the photo-excited electrons to the films surface, and (iii) escape of these electrons to the vacuum by surface tunneling. We tested if the step (i) was limiting the QE of the perovskite thin films by calculating the internal quantum efficiency defined as the QE divided by the absorbance (Fig. 1c and Fig. 2a). It yielded nearly an identical response as the QE in all types of perovskites (Fig. 3b and Supplementary Fig. 2), thus implying the process of photoexcitation of electron carriers in the perovskite thin films is not the main limiting factor of the QE. The efficiency of the step (ii) is largely determined by the transport properties of the perovskite films. After photoexcitation the charge carriers in the films are subject to an electric field of about $3.6 \mathrm{~V} / \mathrm{cm}$ and the efficiency of charge transport to the halide perovskite surface is quantified by the mobility-lifetime product $(\mu \tau)$. In halide perovskites, $\mu \tau$ is typically between $10^{-2}$ and $10^{-4} \mathrm{~cm}^{2} / \mathrm{V}$ depending on the material composition, which is on par or better than III-V semiconductors $8,28,32,33$. Moreover, transport of charge carriers in the bulk of $3 \mathrm{D}$ perovskite thin films is very efficient across a distance of $500 \mathrm{~nm}^{10,11}$. Therefore, we infer that in $3 \mathrm{D}$ halide perovskites the QE of the photoelectric effect is mainly limited by the step (iii), which is the efficiency of ejecting the photogenerated electrons out of the surface. The efficiency of step (iii) is limited by the recombination of electrons at surface defects and surface energy barriers for electrons ${ }^{34}$. The probability of surface recombination depends on the surface recombination velocity $S$, which needs to be minimized in order to achieve high QE-for example smaller than $10^{4} \mathrm{~cm} / \mathrm{s}$ in GaAs electron sources ${ }^{35}$. Previous reports have claimed $S$ values of the order of $10^{3}-10^{4} \mathrm{~cm} / \mathrm{s}$ in $\mathrm{CsPbBr}_{3}$ single crystal ${ }^{36}$, and values as low as $4 \mathrm{~cm} / \mathrm{s}$ in $\mathrm{MAPbBr}_{3}$ single crystals under specific passivation conditions ${ }^{37}$. However, polycrystalline films might have significantly higher $S$ due to the presence of grain boundaries and a higher density of surface defect states as compared to single crystals in the absence of any surface treatment. Indeed, in the $\mathrm{FA}_{0.7} \mathrm{MA}_{0.25} \mathrm{Cs}_{0.05} \mathrm{PbI}_{3}$ thin films, we recently demonstrated that charge collection in solar cells is limited by interface defects, which factors into the lower QE observed in these films ${ }^{28}$. In addition to these effects, in 2D Ruddlesden-Popper layered perovskites less effective transport of the photo-excited electrons to the film surface $\left(\mu \tau \sim 10^{-6}-10^{-5} \mathrm{~cm}^{2} / \mathrm{V}\right)$ can also contribute to the observed lower QE, as explained in our recent work on 2D perovskite photovoltaic devices demonstrating field-dependent charge collection possibly limiting electron-hole separation ${ }^{38}$.

To verify that in $3 \mathrm{D}$ perovskites the $\mathrm{QE}$ is limited by the ability of electrons to escape from the perovskite surface and into the vacuum, we elucidate the impact of the Cs deposition on the emission of free electrons. The minimum photon energy for electron emission in vacuum corresponds to the work function $\mathrm{h} v=\mathrm{W}$, where $h$ is the Plank constant, $v$ is photon frequency, and $W$ the work function. The work functions values reported in the present paper for all pristine perovskites (around $4.0 \mathrm{eV}$ ), including $\mathrm{CsPbBr}_{3}$ (vide infra) are consistent with the work function of $\mathrm{CsPbBr}_{3}$ thin films reported in a recent study at $4.1 \mathrm{eV}^{39}$. We may notice that these values differ from the bulk ionization energies $\mathrm{IE}=E_{\text {gap }}+\chi$, where $E_{\text {gap }}$ is the bandgap and $\chi$ is the electron affinity, defined as the difference between vacuum level and conduction band minimum. Indeed, the ionization energy was measured to be around $5.8 \mathrm{eV}$ in $\mathrm{CsPbBr}_{3}$ thin films ${ }^{39}$. Consistently, the pristine $\mathrm{CsPbBr}_{3}$ thin films in the present work yield an emission onset of $4.0 \mathrm{eV}$, far above the expected bulk ionization energy. Another surprising observation is that the photoelectron onset at $4.0 \mathrm{eV}$ is also common to $\mathrm{CsPbI}_{3}$ and the three hybrid perovskites that were tested, before Cs activation (Fig. 2a and Supplementary Fig. 1). This result is consistent with recent reports claiming negligible dependence of the work function of perovskite thin films as a function of their 
a

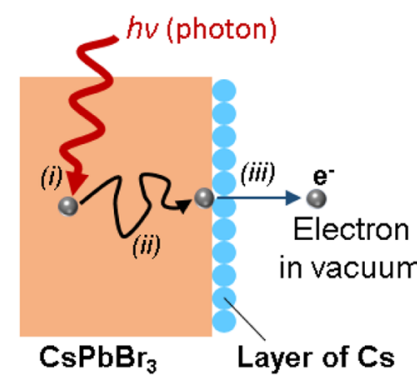

C
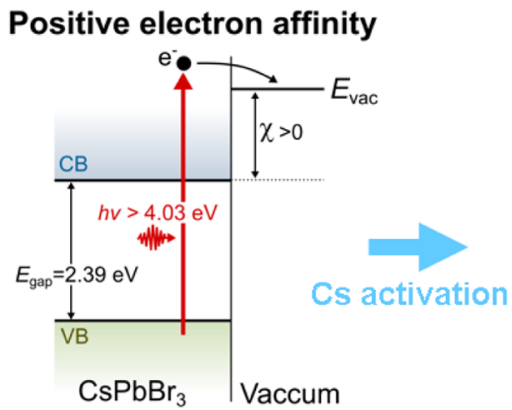

d

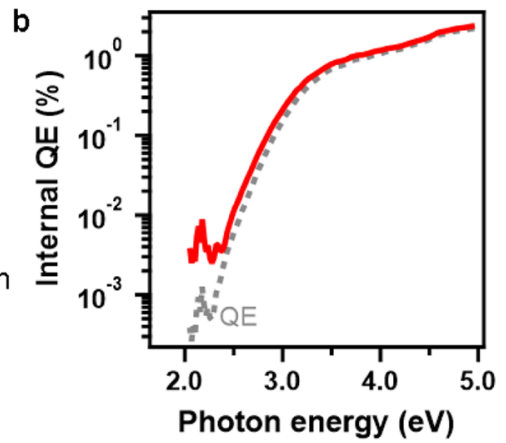

Negative electron affinity

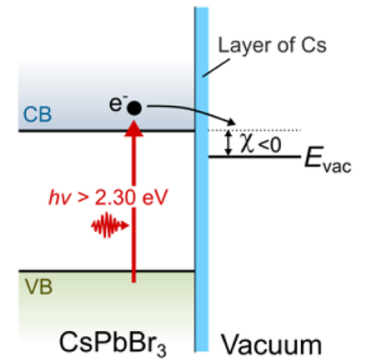

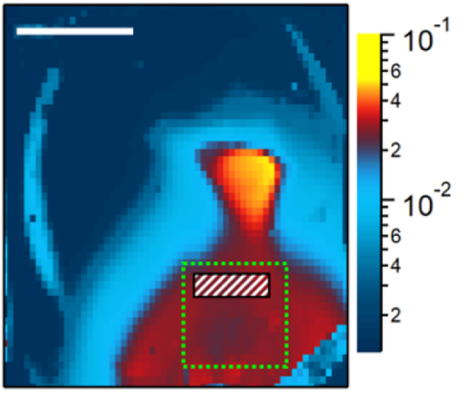

号

Fig. 3 Physical origin of the efficiency of the photoelectric effect. a Mechanism of the photoelectric effect showing the three main steps: (i) electron photogeneration, (ii) electron transport, and (iii) electron emission. $\mathbf{b}$ Internal quantum efficiency of the photoelectric effect in the $\mathrm{CsPbBr} 3$ thin film after Cs activation. The gray dashed curve shows the corresponding (external) quantum efficiency, copied from Fig. 2a. c Schematics of the band diagram at the $\mathrm{CsPbBr} 3$ thin film surface before and after $\mathrm{Cs}$ activation. Egap, VB, CB, $\chi$ stand for the bandgap, the valence band, the conduction band, and electron affinity. Evac indicates the vacuum level where the electron can be emitted from the surface after photoexcitation (hv). d Spatial map of the quantum efficiency relative to its maximum value for the $\mathrm{CsPbBr} 3$ thin film after $\mathrm{Cs}$ activation. Scale bar is $10 \mathrm{~mm}$. Dashed area mark the position of the in-situ $\mathrm{Cs}$ source. The quantum efficiency exhibits peak values near the center of the sample in a $10 \mathrm{~mm}^{2}$ area, while other regions of the samples yield slightly smaller but uniform efficiency within $10 \%$ margin variations over a $1 \mathrm{~cm}^{2}$ (indicated by dashed-green-square region).

compositions due to pinning of the Fermi energy, which has been explained by the similar nature of surface states in all perovskite films ${ }^{39}$. The good correspondence between the $\mathrm{CsPbBr}_{3}$ work function and the electron emission onset suggests that the activation of deep surface traps under vacuum conditions is related to the metal cation $\mathrm{Pb}$ at the film surface ${ }^{37}$. We explain the orders of magnitude increase of the QE upon Cs surface deposition by a conditioning of the thin film surfaces resulting in a change of sign of the electron affinity (Fig. 3c) ${ }^{34}$. Upon deposition of Cs on the perovskite surface, the Cs lose an electron, resulting in the formation of a surface dipole layer that lowers the electron affinity to a negative value ${ }^{14,18,23,40}$. Correspondingly, first-principles calculations explain the increase of QE upon the addition of a Cs layer onto perovskite crystals by a decrease of the work function, which may result from changes in the Fermi energy and density of states at the conduction band minimum (see section ST1 of the supplementary materials and Supplementary Fig. 3). Even though requirements for surface conditioning are relaxed in halide perovskites in comparison with III-V semiconductors ${ }^{18,24}$, both the inhomogeneous spatial distribution of QE (Fig. 3d) and the significantly different QE values observed in the three types of $3 \mathrm{D}$ perovskite films underscore the limited understanding and control of the Cs activation process in halide perovskites and is the main limiting factor for achieving higher QE.

To better understand the relationship between Cs coverage on the halide perovskite surface and QE, we performed in-situ AES measurements. The Cs coverage is quantitatively determined by calculating the ratio between $\mathrm{Cs}(49 \mathrm{eV})$ and $\mathrm{Br}(55 \mathrm{eV})$ Auger peaks (see section ST2) and plotted as a function of QE at $405 \mathrm{~nm}$ in Supplementary Fig. 8b. The QE continuously increased as Cs coverage increased from 0 till about $2.5 \mathrm{Cs} /$ unit cell where QE 


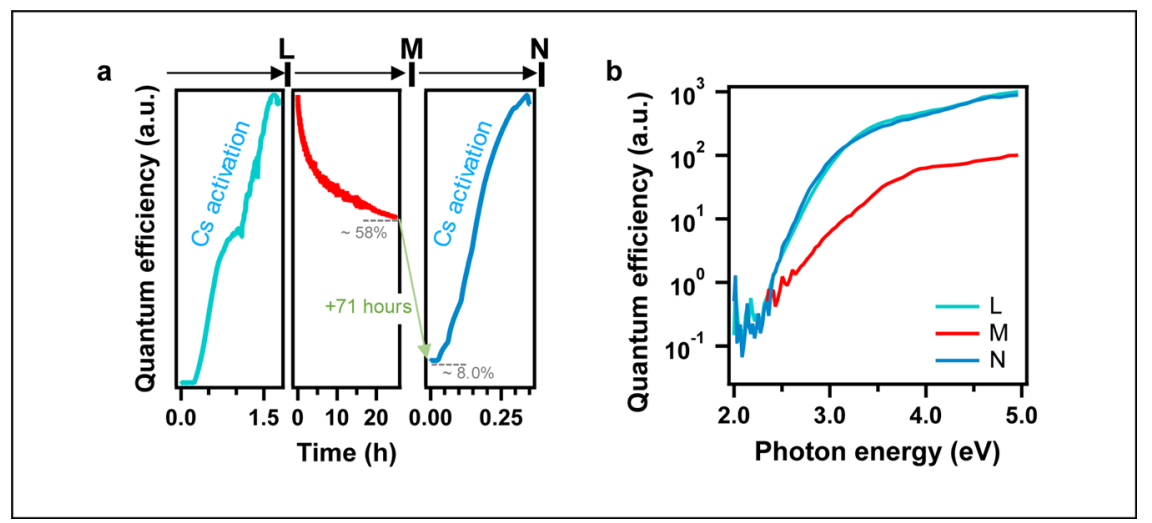

Fig. 4 Stability of the photoelectric effect. a Time evolution of the quantum efficiency of the photoelectric effect in the $\mathrm{CsPbBr} 3$ thin films measured for continuous illumination at $3.06 \mathrm{eV}$ and base vacuum of $10^{-9}$ torr. The left and right sub-plot correspond to $\mathrm{Cs}$ activation processes during which we expose the films to the Cs source and at the same time monitor the quantum efficiency (see also Supplementary Fig. 6). The central sub-plot probes monitor the efficiency degradation over $25 \mathrm{~h}$. The illumination was kept until $96 \mathrm{~h}$ when the second $\mathrm{Cs}$ activation was performed. b Corresponding quantum efficiency spectra were taken at points $L, M$, and $N$.

reached its maximum value. This is in good agreement with our theoretical calculations, which show that two layers of Cs coating (2 Cs/unit cell) has a lower work function than one layer of Cs coating. Furthermore, deposition of more than two layers of Cs decreases the QE, similar to what has been observed on GaAs photocathodes ${ }^{41}$.

Stability of the photoelectric effect during halide perovskite electron source operation. Next, we investigated the stability of the photoelectric effect in the $\mathrm{CsPbBr}_{3}$ thin films. Figure $4 \mathrm{a}$ (central panel) illustrates the evolution of the QE over time as a film is continuously illuminated with light at $3.06 \mathrm{eV}$. We observed that the QE degrades to $60 \%$ of its original value after $25 \mathrm{~h}$ and down to $8 \%$ after $96 \mathrm{~h}$. The main degradation mechanism is attributed to the surface contamination due to the presence of oxygen-based elements inside the vacuum chamber. We quantify the stability of the perovskite films by calculating the exposure in units of Langmuir (L) equivalent to the dose of a given element for the QE to decrease by $63 \%$. We estimated the minimum values of $2.86 \mathrm{~L}, 6.75 \mathrm{~L}$, and $6.28 \mathrm{~L}$ for $\mathrm{H}_{2} \mathrm{O}, \mathrm{O}_{2}$, and $\mathrm{CO}_{2}$, respectively. Under similar conditions of vacuum pressure as used here $\left(10^{-9}\right.$ torr $)$, state-of-the-art semiconductor electron sources yield lower exposures with rare exceptions such as $\mathrm{GaN}^{22,25,42}$. For example, about $0.05 \mathrm{~L}$ for $\mathrm{H}_{2} \mathrm{O}$ was found in a $\mathrm{CsK}_{2} \mathrm{Sb}$ electron source, and exposures smaller than $0.08 \mathrm{~L}$ were reported in a GaAs electron source with an initial QE of $12 \% 18,25$.

Finally, we observed that after degradation the QE of the perovskite thin films could be regenerated back to its original value (i.e. before degradation) via in-situ Cs deposition in about $1 \mathrm{~h}$. Figure 4a illustrates a complete cycle starting from an initial Cs activation (left panel where the QE is continuously monitored while the films are exposed to the in-situ Cs source), followed by a decrease in the QE due to degradation during operation (central panel), and then a second Cs activation process that regenerates the perovskite thin films (right panel). The corresponding QE spectrum of the photoelectric effect taken at different time of the degradation-regeneration cycles confirms that the thin film is almost perfectly regenerated to its original $\mathrm{QE}$ value after the second Cs activation (Fig. 4b). It was demonstrated that the main degradation mechanism in semiconducting GaAs electron sources results from the alteration of the Cs activation layer on the surface. Indeed, exposition of the Cs surface to oxygen-based residual gas in the vacuum chamber leads to the formation of $\mathrm{Cs}-\mathrm{O}$ and $\mathrm{Cs}-\mathrm{OH}$ compounds, thus compromising the beneficial effect of Cs on the performances of the electron sources ${ }^{26,43}$.
Our observation indicates no appreciable degradation of the halide perovskite films as, after degradation, we can almost fully restore the QE of our electron sources through a second Cs activation (Fig. 4a). Therefore, it is reasonable to assume that the main degradation in our electron sources takes place at the Cs surface, most likely through a mechanism similar to the one described in GaAs electron sources. The in-situ AES measurements show the Cs coverage decreased from $2.5 \mathrm{Cs} /$ unit cell (maximum QE) to $2.1 \mathrm{Cs} / \mathrm{unit}$ cell after degradation (Supplementary Fig. 8b) while the relative oxygen Auger peak increased by $\sim 30 \%$ (Supplementary Fig. 9). After adding fresh Cs on the degraded photocathode, the Cs coverage increased to $2.7 \mathrm{Cs} /$ unit cell and the oxygen peak decreased to a magnitude similar to that of the originally activated surface. Moreover, we observe in Fig. 4a (left and right panels) that the Cs-activation process after degradation is about four times faster than the initial Csactivation (from the pristine films), which is another indication that the Cs surface is only partially compromised by the oxygenbased residual gas and needs to be restored with Cs atoms.

In summary, we have demonstrated perovskite-based electron sources with a few percent quantum efficiencies, with spectral tunability other than the visible spectral range, and which can be operated for tens of hours and integrally regenerated in situ after degradation. The figures of merit reported for the photoelectric effect in halide perovskite thin films offer a tremendous opportunity to develop a disruptive electron source technology that addresses the demands for low-cost fabrication and operation, high efficiency, and spectral tunability. Furthermore, future optimization of the quality of the perovskite thin films and the Cs deposition process should lead to electron sources preforming on par with those obtained using III-V semiconductors but with orders of magnitude lower cost in terms of manufacturing and operation.

\section{Methods}

Thin film samples preparation. All chemicals, lead bromide $\left(\mathrm{PbBr}_{2}\right)$, lead iodide $\left(\mathrm{PbI}_{2}\right)$, cesium bromide (CsBr), cesium iodide (CsI), N, N-dimethylformamide (DMF), dimethyl sulfoxide (DMSO), formamidinium iodide (FAI), methylammonium iodide (MAI), butylammine (BA) were purchased from Sigma-Aldrich with the purity of $99 \%$ or higher. Equal molar of $\mathrm{PbBr}_{2}$ and $\mathrm{CsPb}$ were mixed in DMSO for $0.4 \mathrm{M} \mathrm{CsPbBr}_{3}$ solution. Equal molar of $\mathrm{PbI}_{2}$ and CsI was mixed in DMF for $0.6 \mathrm{M} \mathrm{CsPbI}_{3}$ solution. Both solutions were stirred for $24 \mathrm{~h}$. The

Ruddlelden-Popper layered perovskite solutions were prepared by dissolving the layered perovskite single crystals in DMF with $0.255 \mathrm{M}$ molar concentration. The Ruddlesden-Popper layered perovskite single crystals were synthesized following a method reported previously ${ }^{44-47}$. $\mathrm{PbO}$ powder was dissolved in a mixture of $57 \%$ w/w aqueous $\mathrm{HI}$ solution and $50 \%$ aqueous $\mathrm{H}_{3} \mathrm{PO}_{2}$ by heating to boiling under 
constant magnetic stirring. An appropriate molar amount of MAI and BA were added to the hot solution separately. The solution was left to cool to room temperature while the perovskite crystals started to precipitate. $\mathrm{FA}_{0.7} \mathrm{MA}_{0.25} \mathrm{Cs}_{0.05} \mathrm{PbI}_{3}$ solution was a mix of $\mathrm{PbI}_{2}, \mathrm{FAI}, \mathrm{MAI}$, and CsI with the desired molar ratio and dissolved in DMSO with a molar concentration of $0.43 \mathrm{M}^{28}$. Doped silicon substrates were cut into $1 \mathrm{inch}^{2}$ and treated by oxygen plasma before spin coating. The substrates resistivity was chosen between 0.001 and $0.005 \Omega \cdot \mathrm{cm}$ and their thickness about $500 \mu \mathrm{m}$, in order to prevent any performance limitation due to the substrates. The prepared solutions were spin coated onto the Si substrates with a speed $2000 \mathrm{rpm}$ for $\mathrm{CsPbBr}_{3}$ and $3000 \mathrm{rpm}$ for $\mathrm{CsPbI}_{3}$ in order to get $\sim 200 \mathrm{~nm}$ thick films. The films were then annealed on a hot plate at $100^{\circ} \mathrm{C}$ for $10 \mathrm{~min}$. The $2 \mathrm{D}$ perovskite and $\mathrm{FA}_{0.7} \mathrm{MA}_{0.25} \mathrm{Cs}_{0.05} \mathrm{PbI}_{3}$ films were prepared by hot casting method $^{29,47}$. All processes were conducted in an Ar filled glovebox except for substrate preparation. The samples were transported in a sealed vial filled with argon and loaded into the vacuum chamber load lock with minimum exposure to air (less than $5 \mathrm{~min}$ ). Inside the load lock, only the $\mathrm{CsPbBr}_{3}$ and $\mathrm{CsPbI}_{3}$ thin films were baked out at $350{ }^{\circ} \mathrm{C}$ for $24 \mathrm{~h}$ to clean the solvent residue and absorbed moistures; the hybrid halide perovskite films were not backed to prevent degradation due to temperature. After the samples were cooled down, they were loaded into the main chamber with a base pressure of about $10^{-9}$ torr and with residual gases partial pressures smaller than $10^{-10}$ torr $\left(\mathrm{H}_{2} \mathrm{O} 1.59 \times 10^{-11}\right.$ torr, $\mathrm{O}_{2} 3.75 \times$ $10^{-11}$ torr, $\mathrm{CO}_{2} 3.49 \times 10^{-11}$ torr). The vacuum chamber was analyzed by a Stanford Research System Residual Gas Analyzer.

Photoelectric effect measurement. For photoexcitation of the thin films inside the vacuum chamber, we used the monochromatic light generated by a Newport $150 \mathrm{~W}$ Xenon lamp coupled with a monochromator equipped with a $300 \mathrm{l} / \mathrm{mm}$ grating and long-pass filters; the range of monochromatic light available for the QE spectra was 4.96 to $1.55 \mathrm{eV}(250$ to $800 \mathrm{~nm})$. The light was modulated at $400 \mathrm{~Hz}$ by a chopper and then directed into the chamber by a mirror and through a quartz window at $45^{\circ}$ incident angle. The light beam was illuminating about $10 \mathrm{~mm}^{2}$ of the thin film surface if not mentioned otherwise, and the light power at the sample position was in the range $38-50 \mu \mathrm{W}$. In this regime, the QE of the films does not show appreciable changes as a function of light intensity, in order to keep surface charging effects at a minimum (Supplementary Fig. 5). For mapping of the QE, the light beam from a 405-nm laser was scanned onto the thin film surface using a stirring mirror and the photocurrent was acquired at each scanned point. The anode was placed $25 \mathrm{~mm}$ away from the film substrates, and biased at $+90 \mathrm{~V}$ against the doped Si substrate using DC batteries in order to minimize electronic noise. The chamber was connected to the anode to eliminate any possible chamber emission. In our setup, $90 \mathrm{~V}$ was tested to be high enough to collect all photoelectrons (Supplementary Fig. 4). A $100 \mathrm{k} \Omega$ resister was connected in series with the cathode to convert the photocurrent into voltage. The circuit capacitance was $2.7 \mathrm{nF}$, corresponding to a time constant of $0.27 \mathrm{~ms}$, much faster than the light modulation period $(2.5 \mathrm{~ms})$. The voltage was measured by an SR830 lock-in amplifier with a time constant between $0.3 \mathrm{~s}$ and $10 \mathrm{~s}$ depending on the magnitude of the signal. All measurements were performed at ambient temperature.

Cs deposition. For the process of Cs activation, we applied current to a $1 \mathrm{~cm}$ long SAES Cs dispenser as a Cs deposition source. Prior to deposition, the source was first degassed in an antechamber at a current of $4 \mathrm{~A}$. The source was then placed in front of the sample with a $2.5-5 \mathrm{~cm}$ distance. The light source was placed at $405 \mathrm{~nm}$ to monitor the photocurrent from the photoelectric effect. The current was slowly ramped up at a rate of $0.1 \mathrm{~A} / \mathrm{min}$ until the photocurrent started to increase, typically around 4.5 to $5 \mathrm{~A}$. The source current was either maintained or further increased to maximize the photocurrent, just before the photocurrent started to drop meaning in that case that the deposited layer of Cs onto the thin film surface was too thick (Supplementary Fig. 6). The Cs source was then retracted from the deposition position and turned off. An energy-dependent scan was performed immediately after the Cs deposition to measure the QE spectrum. From our observations, it is reasonable to assume that the Cs layer on the film's surface is limited to at most a few monolayers of Cs and its thickness is relatively inhomogeneous due to the roughness of the films. This is supported by several evidences: the lack of electron emission from the Cs layer, the low sticking coefficient between $\mathrm{Cs}$ atoms and the low melting temperature $\left(28.4^{\circ} \mathrm{C}\right)$, and the rapid degradation of the $\mathrm{QE}$ of halide perovskite sources for thicker Cs layers (Supplementary Fig. 6) consistent with observations reported in Cs activated GaAs electron sources.

Auger electron spectroscopy. The Auger spectra were obtained with a SPECS Phoibos 150 hemispherical energy analyzer and a five channel multi channeltron detector (MCD-5). The electron gun was set at $3 \mathrm{keV}$ energy and $1 \mu \mathrm{A}$ current. The area of the electron beam was $\sim 1 \mathrm{~mm}^{2}$. The AES UHV chamber was connected to the Cs deposition chamber through a sample transfer arm.

\section{Data availability}

The data that support this study within the paper and supplementary materials are available from the corresponding authors upon request.
Received: 9 March 2020; Accepted: 4 January 2021; Published online: 29 January 2021

\section{References}

1. Correa-Baena, J.-P. et al. Promises and challenges of perovskite solar cells. Science 358, 739-744 (2017).

2. Zhang, W., Eperon, G. E. \& Snaith, H. J. Metal halide perovskites for energy applications. Nat. Energy 1, 1-8 (2016).

3. Best Research-Cell Efficiency Chart. Photovoltaic Research (NREL). https:// www.nrel.gov/pv/cell-efficiency.html.

4. Snaith, H. J. Present status and future prospects of perovskite photovoltaics. Nat. Mater. 17, 372-376 (2018).

5. Yuan, M. et al. Perovskite energy funnels for efficient light-emitting diodes. Nat. Nanotechnol. 11, 872-877 (2016).

6. Kovalenko, M. V., Protesescu, L. \& Bodnarchuk, M. I. Properties and potential optoelectronic applications of lead halide perovskite nanocrystals. Science $\mathbf{3 5 8}$ 745-750 (2017).

7. Kim, Y. C. et al. Printable organometallic perovskite enables large-area, lowdose X-ray imaging. Nature 550, 87-91 (2017).

8. He, Y. et al. High spectral resolution of gamma-rays at room temperature by perovskite CsPbBr 3 single crystals. Nat. Commun. 9, 1609 (2018).

9. Chen, Q. et al. All-inorganic perovskite nanocrystal scintillators. Nature 561, 88-93 (2018)

10. Stranks, S. D. et al. Electron-hole diffusion lengths exceeding 1 micrometer in an organometal trihalide perovskite absorber. Science 342, 341-344 (2013).

11. Shi, D. et al. Low trap-state density and long carrier diffusion in organolead trihalide perovskite single crystals. Science 347, 519-522 (2015).

12. Herz, L. M. Charge-carrier dynamics in organic-inorganic metal halide perovskites. Annu. Rev. Phys. Chem. 67, 65-89 (2016).

13. Egger, D. A. et al. What remains unexplained about the properties of halide perovskites? Adv. Mater. 30, 1800691 (2018).

14. Bell, R. L. Negative Electron Affinity Devices (Clarendon Press, 1973).

15. Williams, B. F. \& Tietjen, J. J. Current status of negative electron affinity devices. Proc. IEEE 59, 1489-1497 (1971).

16. Baum, A. W., Schneider, J. E. \& Pease, R. F. W. High-performance negative electron affinity photocathodes for high resolution electron beam lithography and metrology. in Proceedings of International Electron Devices Meeting 409-412 (1995). https://doi.org/10.1109/IEDM.1995.499226.

17. Roaux, E., Richard, J. C. \& Piaget, C. in Advances in Electronics and Electron Physics (ed. Morgan, B. L.) Vol. 64, 71-75 (Academic Press, 1986).

18. Rao, T. \& Dowell, D. H. An Engineering Guide to Photoinjectors (Createspace Independent Pub, 2013).

19. Srinivasan-Rao, T., Fischer, J. \& Tsang, T. Photoemission studies on metals using picosecond ultraviolet laser pulses. J. Appl. Phys. 69, 3291-3296 (1991)

20. Moody, N. A. et al. Perspectives on designer photocathodes for X-ray freeelectron lasers: influencing emission properties with heterostructures and nanoengineered electronic states. Phys. Rev. Appl. 10, 047002 (2018).

21. Gaowei, M. et al. Codeposition of ultrasmooth and high quantum efficiency cesium telluride photocathodes. Phys. Rev. Accel. Beams 22, 073401 (2019).

22. Siegmund, O. et al. Development of GaN photocathodes for UV detectors. Nucl. Instrum. Methods Phys. Res. Sec. A 567, 89-92 (2006).

23. Turnbull, A. A. \& Evans, G. B. Photoemission from GaAs-Cs-O. J. Phys. D 1, 155-160 (1968).

24. Liu, Z. Surface Characterization of Semiconductor Photocathode Structures (PhDT, 2005)

25. Chanlek, N. et al. The degradation of quantum efficiency in negative electron affinity GaAs photocathodes under gas exposure. J. Phys. D 47, 055110 (2014).

26. Wada, T., Nitta, T., Nomura, T., Miyao, M. \& Hagino, M. Influence of Exposure to CO, $\mathrm{CO}_{2}$ and $\mathrm{H}_{2} \mathrm{O}$ on the Stability of GaAs Photocathodes. Jpn. J. Appl. Phys. 29, 2087 (1990).

27. Durek, D., Frommberger, F., Reichelt, T. \& Westermann, M. Degradation of a gallium-arsenide photoemitting NEA surface by water vapour. Appl. Surf. Sci. 143, 319-322 (1999).

28. Tsai, H. et al. Light-induced lattice expansion leads to high-efficiency perovskite solar cells. Science 360, 67-70 (2018).

29. Tsai, H. et al. High-efficiency two-dimensional Ruddlesden-Popper perovskite solar cells. Nature 536, 312-316 (2016).

30. Juarez-Perez, E. J. et al. Photodecomposition and thermal decomposition in methylammonium halide lead perovskites and inferred design principles to increase photovoltaic device stability. J. Mater. Chem. A 6, 9604-9612 (2018).

31. Blancon, J.-C. et al. Extremely efficient internal exciton dissociation through edge states in layered 2D perovskites. Science 355, 1288-1292 (2017).

32. Wei, H. \& Huang, J. Halide lead perovskites for ionizing radiation detection. Nat. Commun. 10, 1-12 (2019). 
33. Hutter, E. M. et al. Vapour-deposited cesium lead iodide perovskites: microsecond charge carrier lifetimes and enhanced photovoltaic performance. ACS Energy Lett. 2, 1901-1908 (2017).

34. Schulz, P., Cahen, D. \& Kahn, A. Halide perovskites: is it all about the interfaces? Chem. Rev. 119, 3349-3417 (2019).

35. Fu, J.-T., Zheng, G.-Q. \& Zhang, S.-C. Study on the influence of recombination velocity to quantum efficiency of reflection-mode GaAs photocathode. Optik 124, 1862-1864 (2013).

36. Zhu, H. et al. Organic cations might not be essential to the remarkable properties of band edge carriers in lead halide perovskites. Adv. Mater. 29, 1603072 (2017).

37. Fang, H.-H. et al. Ultrahigh sensitivity of methylammonium lead tribromide perovskite single crystals to environmental gases. Sci. Adv. 2, e1600534 (2016).

38. Tsai, H. et al. Design principles for electronic charge transport in solutionprocessed vertically stacked 2D perovskite quantum wells. Nat. Commun. 9, 2130 (2018).

39. Endres, J. et al. Valence and conduction band densities of states of metal halide perovskites: a combined experimental-theoretical study. J. Phys. Chem. Lett. 7, 2722-2729 (2016).

40. Kahn, A. Fermi level, work function and vacuum level. Mater. Horiz. 3, 7-10 (2015).

41. Kierren, B. \& Paget, D. Formation of the Cs/GaAs (001) interface: work function, cesium sticking coefficient, and surface optical anisotropy. J. Vac. Sci. Technol. A 15, 2074-2080 (1997).

42. Chanlek, N. et al. High stability of negative electron affinity gallium arsenide photocathodes activated with Cs and NF3. J. Phys. D 48, 375102 (2015).

43. Rodway, D. C. \& Allenson, M. B. In situ surface study of the activating layer on GaAs (Cs, O) photocathodes. J. Phys. D 19, 1353-1371 (1986).

44. Cao, D. H., Stoumpos, C. C., Farha, O. K., Hupp, J. T. \& Kanatzidis, M. G. 2D homologous perovskites as light-absorbing materials for solar cell applications. J. Am. Chem. Soc. 137, 7843-7850 (2015).

45. Stoumpos, C. C. et al. Ruddlesden-popper hybrid lead iodide perovskite 2D homologous semiconductors. Chem. Mater. 28, 2852-2867 (2016).

46. Stoumpos, C. C. et al. High members of the 2D Ruddlesden-Popper halide perovskites: synthesis, optical properties, and solar cells of $\left(\mathrm{CH}_{3}\left(\mathrm{CH}_{2}\right)_{3} \mathrm{NH}_{3}\right)_{2}\left(\mathrm{CH}_{3} \mathrm{NH}_{3}\right)_{4} \mathrm{~Pb}_{5} \mathrm{I}_{16}$. Chem 2, 427-440 (2017).

47. Nie, W. et al. High-efficiency solution-processed perovskite solar cells with millimeter-scale grains. Science 347, 522-525 (2015).

\section{Acknowledgements}

The work at Rice University was supported by ARO STIR project N-mensional interfaces, grant W911NF-19-1-0353. The work at Los Alamos National Laboratory (LANL) was supported by the LANL Laboratory Directed Research and Development Funds (LDRD) program. This work was done in part at the Center for Nonlinear Studies (CNLS) and the Center for Integrated Nanotechnologies (CINT), a U.S. Department of Energy and Office of Basic Energy Sciences user facility, at LANL. This research used resources provided by the LANL Institutional Computing Program. Los Alamos National Laboratory is operated by Triad National Security, LLC, for the National Nuclear Security Administration of the U.S. Department of Energy (Contract No. 89233218NCA000001).
J.E. acknowledges the financial support from the Institut Universitaire de France. Work at Northwestern was supported by the U.S. Department of Energy, Office of Science (Grant No. SC0012541, structure characterization). We thank Anna Marie Alexander and Anju Poudel for their help with Auger electron spectroscopy.

\section{Author contributions}

J.-C.B. and A.D.M. conceived the idea and designed the experiment. F.L. synthesized the perovskite thin films, characterized the materials, and performed the photoemission experiments. H.T., W.N., and S.S. advised. F.L. on the synthesis of the $3 \mathrm{D}$ and $2 \mathrm{D}$ hybrid perovskite films. F.L., M.A.H., V.P., and N.A.M. built the UHV system. S.L., A.J.N., J.E., and S.T. performed the DFT calculation. S.S., P.M.A., M.G.K., and J.J.C. helped with data analysis. F.L., J.-C.B., and A.D.M. wrote the manuscript with inputs from everyone. All authors read the manuscript and agree to its contents, and all data are reported in the main text and supplemental materials. The authors declare that the main data supporting the findings of this study are available within the article and its Supplementary information.

\section{Competing interests}

The authors declare no competing interests.

\section{Additional information}

Supplementary information The online version contains supplementary material available at https://doi.org/10.1038/s41467-021-20954-6.

Correspondence and requests for materials should be addressed to J.-C.B. or A.D.M.

Peer review information Nature Communications thanks the anonymous reviewer(s) for their contribution to the peer review of this work.

Reprints and permission information is available at http://www.nature.com/reprints

Publisher's note Springer Nature remains neutral with regard to jurisdictional claims in published maps and institutional affiliations.

\begin{abstract}
cc (i) Open Access This article is licensed under a Creative Common BY Attribution 4.0 International License, which permits use, sharing adaptation, distribution and reproduction in any medium or format, as long as you give appropriate credit to the original author(s) and the source, provide a link to the Creative Commons license, and indicate if changes were made. The images or other third party material in this article are included in the article's Creative Commons license, unles indicated otherwise in a credit line to the material. If material is not included in the article's Creative Commons license and your intended use is not permitted by statutory regulation or exceeds the permitted use, you will need to obtain permission directly from the copyright holder. To view a copy of this license, visit http://creativecommons.org/ licenses/by/4.0/.
\end{abstract}

(c) The Author(s) 2021 